\title{
VARIAÇÃO DA BIOMASSA AÉREA DE Allagoptera arenaria (GOMES) O. KUNTZE (ARECACEAE) EM UMA COMUNIDADE ARBUSTIVA DE PALMAE NA RESTINGA DE MARAMBAIA, RJ
}

\author{
MENEZES, L. F. T. de e ARAUJO, D. S. D. de ${ }^{2}$ \\ ${ }^{1}$ Departamento de Botânica, Instituto de Biologia, Universidade Federal Rural do Rio de Janeiro, Antiga \\ Rodovia Rio-São Paulo, Km 47, CEP 23851-970, Seropédica, RJ \\ ${ }^{2}$ Departamento de Ecologia, PPGE, Universidade Federal do Rio de Janeiro, Ilha do Fundão, \\ CEP 21941-570, Rio de Janeiro, RJ \\ Correspondência para: Luis Fernando Tavares de Menezes, Departamento de Botânica, Instituto de \\ Biologia, Universidade Federal Rural do Rio de Janeiro, Antiga Rodovia Rio-São Paulo, Km 47, \\ CEP 23851-970, Seropédica, RJ, e-mail: tavares@openlink.com.br \\ Recebido em 13/05/98 - Aceito em 19/03/99 - Distribuído em 28/02/2000.
}

(Com 4 figuras)

\section{ABSTRACT \\ Variation in aboveground biomass of Allagoptera arenaria (Gomes) O. Kuntze (Arecaceae) on the Marambaia beach ridge, Rio de Janeiro, Brazil}

Variation of aboveground biomass of Allagoptera arenaria (Gomes) O. Kuntze (Arecaceae) along five topographic profiles perpendicular to the ocean was examined in a palm scrub community on Marambaia beach ridge, Rio de Janeiro State, Brazil. Aerial biomass was positively correlated with distance from the sea $\left(\mathrm{F}=39.57 ; \mathrm{R}^{2}=0.69 ; \mathrm{P}<0.01\right)$ as was detritus cover $\left(\mathrm{F}=525.92 ; \mathrm{R}^{2}=0.92\right.$; $\mathrm{P}<0.01)$. A. arenaria growth is closely related to the topography of the beach area. Dense populations of this palm enrich the soil by increasing organic matter under the plants through dead leaf material. This promotes the accumulation of nutrients and the creation of microclimates that favor the establishment of other species.

Key words: biomass, Allagoptera arenaria, sandy coastal plain, Rio de Janeiro.

\section{RESUMO}

A variação da biomassa aérea de Allagoptera arenaria (Gomes) O. Kuntze (Arecaceae), ao longo de 5 perfis topográficos perpendiculares ao mar, foi analisada em uma comunidade arbustiva de Palmae, na restinga de Marambaia, RJ. Foi encontrada uma relação positiva e altamente significativa da biomassa aérea dessa espécie $\left(\mathrm{F}=39,57 ; \mathrm{R}^{2}=0,69 ; \mathrm{P}<0,01\right)$ e da cobertura de detritos $\left(\mathrm{F}=525,92 ; \mathrm{R}^{2}=\right.$ $0,92 ; \mathrm{P}<0,01)$ com o distanciamento do mar. O desenvolvimento de A. arenaria está intimamente relacionado com a variação topográfica da área de estudo. Densas populações de $A$. arenaria enriquecem o solo através do aumento de matéria orgânica sob sua cobertura proveniente de suas folhas senescentes e, conseqüentemente, potencializa o acúmulo de nutrientes caracterizando um microclima que pode colaborar no estabelecimento de outras espécies.

Palavras-chave: biomassa, Allagoptera arenaria, restinga, Rio de Janeiro.

\section{INTRODUÇÃo}

Os ecossistemas de restinga caracterizam-se por solos arenosos, pobres em argilas e matéria orgânica, baixa capacidade de reter água e nutrientes e por uma grande proporção da entrada anual de nutrientes sob a forma do salsugem (van der Valk, 1974; Hay e Lacerda, 1984). A baixa quan- 
tidade de nutrientes no solo limita a produção de estruturas vegetativas e reprodutivas, verificandose, paralelamente, um acúmulo e alocação desses nutrientes nas plantas (Schat et al., 1984). Além da falta de nutrientes no solo, que atuam diretamente no desenvolvimento do vegetal, outros fatores assumem particular importância em se tratando de ecossistemas de restinga, influenciando na complexidade das comunidades vegetais. A posição topográfica, por exemplo, pode influenciar algumas características como a diversidade e a estrutura das mesmas (Cordazzo \& Costa, 1989; Cordeiro, 1998). Os desníveis no terreno dão origem a um complexo mosaico intimamente ligado à profundidade do lençol freático (Cordazzo, 1985) e afetam o conteúdo de água no solo, demarcando limites ecologicamente significativos para diferenciar dunas e "slacks" (Packham \& Willis, 1997). O déficit de água nos solos mais elevados contribui para a baixa taxa de crescimento das plantas nestes locais, além de deixá-las mais expostas aos ventos predominantes, afetando sua edificação (Rajpurohit \& Sen, 1977).

Extensas áreas localizadas entre o mar e as dunas, na restinga de Marambaia, caracterizadas por ondulações no terreno formadas por cristas praiais, são recobertas pela comunidade arbustiva de Palmae (Menezes, 1996). Esta comunidade é dominada pela palmeira Allagoptera arenaria. (Gomes) O. Kuntze, que recobre o topo das cristas praiais, assim como o declive entre estas, dando um aspecto homogêneo à paisagem. No litoral sudeste, as áreas de restingas queimadas, principalmente naquelas em que houve sucessivas passagens do fogo, geralmente são dominadas pela $A$. arenaria. (Lacerda et al., 1993). Esta dominância tem sido relacionada a sua reprodução por rizomas (Tomlinson, 1960; Rizzini, 1979; Leite, 1990; Menezes, 1996), a alta capacidade de sobreviver às queimadas (Araujo \& Peixoto, 1977; Almeida \& Araujo, 1997; Cirne \& Scarano, 1996), a disponibilidade de frutos durante todo o ano (Leite, 1990) e a sua forma de vida. Tais características biológicas garantem a essa espécie vantagens na regeneração e colonização de áreas impactadas, onde são formadas moitas densas caracterizando esse tipo peculiar de comunidade vegetal (Almeida \& Araujo, 1997; Cardoso, 1995). A comunidade arbustiva de Palmae foi estudada quanto a sua fitossociologia por Almeida \& Araujo (1997), Cardoso (1995), Menezes (1996) e Menezes \& Araujo (1999). Com este trabalho pretende-se: 1) avaliar o padrão e distribuição da biomassa aérea de $A$. arenaria ao longo do perfil da praia em direção ao continente na restinga de Marambaia; 2) comparar a distribuição de sua biomassa aérea com a distribuição da cobertura de matéria orgânica e nutrientes no solo.

\section{MATERIAL E MÉTODOS}

\section{Descrição da área de estudo}

A restinga de Marambaia é uma longa faixa de areia, com aproximadamente $40 \mathrm{~km}$ de extensão que separa a Baía de Sepetiba do Oceano Atlântico. Localiza-se, em parte, na Zona Oeste do município do Rio de Janeiro e, em parte, nos municípios de Itaguaí e de Mangaratiba (Fig. 1). A temperatura média anual atinge $23,6^{\circ} \mathrm{C}$, sendo o mês de fevereiro mais quente, com temperatura média de $26,7^{\circ} \mathrm{C}$, e julho, mais frio, com temperatura média de $21,0^{\circ} \mathrm{C}$. O total médio anual de precipitação é de 1.027,2 mm, sendo o mês de agosto o mais seco, com uma média de 47,4 mm, e março, o mais chuvoso, com uma média de 140,6 mm (Menezes, 1996). A área de estudo, na extremidade leste da restinga de Marambaia ( $23^{\circ} 03^{\prime} \mathrm{S} ; 44^{\circ} 39^{\prime} \mathrm{W}$ ) é constituída por uma faixa com $3 \mathrm{~km}$ de extensão e 70 a $90 \mathrm{~m}$ de largura. É delimitada ao sul, próximo à praia, pela comunidade psamófilareptante, constituída por espécies de plantas herbáceas, estoloníferas, rizomatosas e reptantes (Menezes, 1996) e ao norte, por uma estrada. A comunidade arbustiva de Palmae, dominada por indivíduos de $A$. arenária com até $1,5 \mathrm{~m}$ de altura, recobre as cristas praiais originárias pelas ondas de tempestades. Essas cristas praiais podem chegar a $4 \mathrm{~m}$ de altura em relação ao ponto zero (localizado na zona entremarés e utilizado como referência para a construção dos perfis topográficos). As depressões entre as cristas praias podem alcançar até 1,5 $\mathrm{m}$ abaixo desse referencial (Menezes, 1996).

\section{Amostragem da vegetação, detritos e solo}

Para a caracterização da variação da biomassa aérea e o desenvolvimento dos indivíduos de Allagoptera arenaria com o distanciamento do mar, foram registradas a altura (distância entre o ponto mais alto da folha, inflorescência ou infrutescência, estendidos até o máximo e a superfície do solo) e o maior eixo (maior comprimento do eixo da copa) de todos os indivíduos com suas bases inseridas em 5 "transect" de $2 \mathrm{~m}$ de largura, perpendiculares ao mar. 


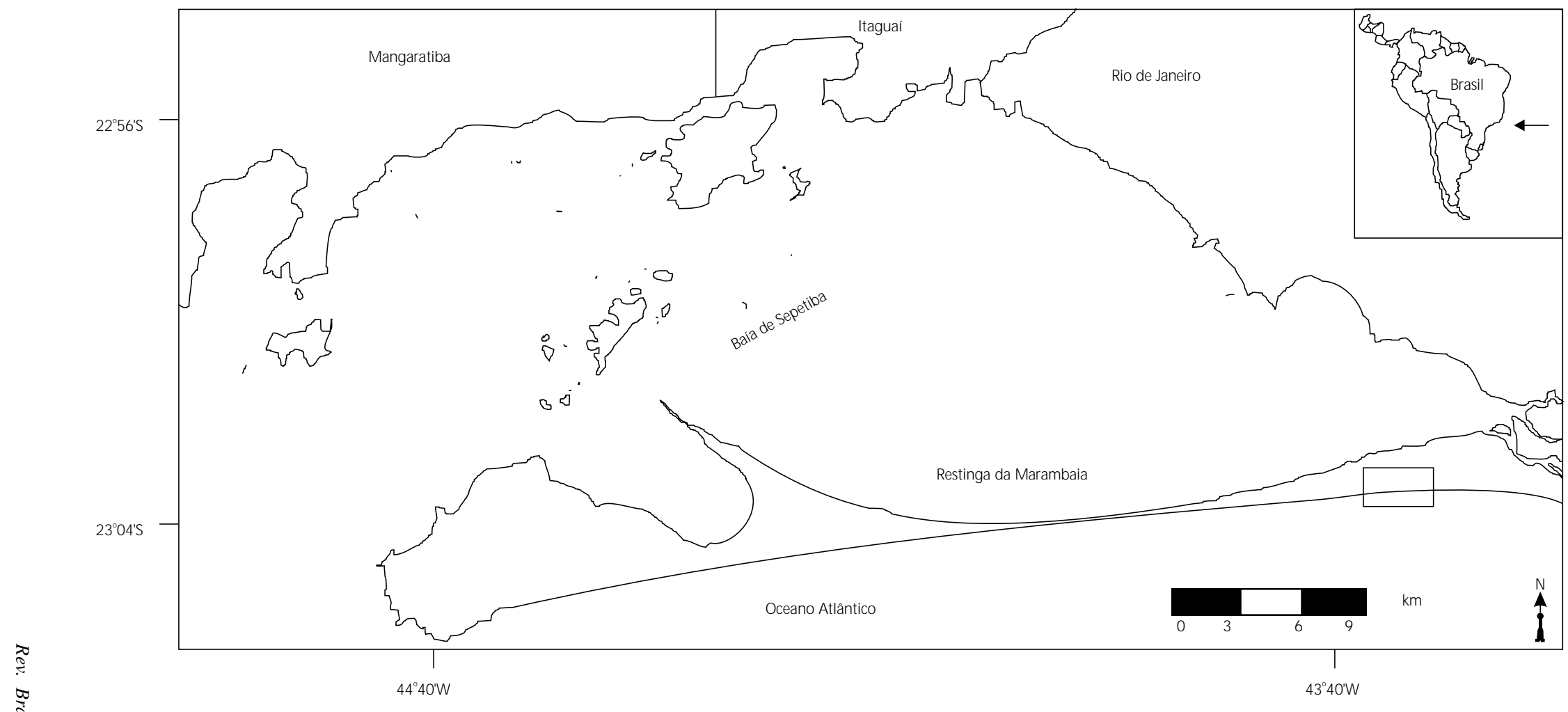

Fig. 1 - Localização da Restinga de Marambaia, no Município do Rio de Janeiro. O retângulo indica a área de estudo. 
Os "transects" foram posicionados no sentido, aproximadamente, norte-sul, e a distância entre cada um variou de 250 a $1.100 \mathrm{~m}$. Os mesmos tiveram seu início onde encontravam-se os primeiros indivíduos de $A$. arenaria, geralmente no limite interno da comunidade psamofila-reptante e estendiam-se até o término da comunidade arbustiva de Palmae, à margem da estrada, perfazendo um total de $700 \mathrm{~m}^{2}$ de área.

Foi considerado como um indivíduo de $A$. arenaria o conjunto de folhas acompanhadas ou não de inflorescências ou infrutescências, isoladas de outros grupos de folhas, independente de estarem ligadas a outros indivíduos por rizoma.

Foi utilizado o diâmetro da copa para a determinação da biomassa aérea por meio da seguinte fórmula proposta por Hay et al. (1982):

$$
y=4,35 e^{2,82 x}
$$

em que $y$ é a biomassa expressa em gramas, $e$ é a constante neperiana e $x$ é o eixo da copa.

O porcentual do substrato coberto por detritos foi estimado no período de 20/08/94 a 28/01/95 por meio de sua projeção vertical, ao longo de 13 linhas perpendiculares ao mar, por parcelas de $1 \mathrm{~m}^{2}$ distribuídas em lados alternados com intervalos de $1 \mathrm{~m}$ entre elas, perfazendo um total de $544 \mathrm{~m}^{2}$ amostrados (Menezes, 1996).

Os perfis topográficos foram os mesmos utilizados por Menezes (1996) para a caracterização de comunidades vegetais praianas no local e coincidiram com os "transects" utilizados para obter os dados de biomassa de $A$. arenaria.

Foram coletadas amostras do solo para análises granulométricas e de fertilidade em três diferentes zonas, localizadas nas linhas onde foram realizados os perfis topográficos. A zona 1 localizou-se no cume da primeira linha de cristas praiais, fora da influência das marés normais, na comunidade psamófila-reptante. As zonas 2 e 3, distantes aproximadamente 30 e $70 \mathrm{~m}$, respectivamente, da zona 1, localizaram-se na comunidade arbustiva de Palmae.

As seis amostras do solo de cada zona foram coletadas a $10 \mathrm{~cm}$ da superfície e, posteriormente, homogeneizadas, formando assim uma única amostra por zona. De cada zona foi retirada uma alíquota de cerca de $400 \mathrm{~g}$ para as análises químicas e físicas. As análises foram realizadas pelo Serviço Nacional de Levantamento e Conservação de Solos da EMBRAPA - RJ.

Para as análises estatísticas dos dados foram verificados a homogeneidade de variância e a normalidade de distribuição de freqüência pelos testes de Cochran \& Bartlett e Lilliefors, respectivamente. As variáveis que não aproximaram-se satisfatoriamente da normalidade e homogeneidade, segundo os testes citados, foram transformadas (indicadas nas tabelas) para uma melhor aproximação. Para os contrastes de médias foi utilizado o teste de Tukey a 5\%. Em todas as análises utilizouse o pacote estatístico SAEG ${ }^{\circledR}$ da Fundação Arthur Bernardes.

\section{RESULTADOS}

Os resultados das análises de fertilidade dos solos (Tabela 1) mostraram um aumento progressivo nas concentrações de $\mathrm{Al}, \mathrm{Ca}+\mathrm{Mg}, \mathrm{Na}, \mathrm{K}, \mathrm{C}$ e acidez no sentido da zona 1 para a 3 (mar-continente). $\mathrm{O}$ inverso foi observado com a concentração de $\mathrm{P}$.

A análise granulométrica dos solos para a área estudada revelou uma alta proporção de areia com porcentagens muito reduzidas de silte e argila (Tabela 1). Ocorreram poucas variações desses componentes em relação à distância do mar.

A Fig. 2 mostra o diagrama esquemático representativo para todos os perfis topográficos realizados na área, com as respectivas zonas para a quantificação da biomassa aérea de $A$. arenaria.

Foram encontrados 859 indivíduos de $A$. arenaria, correspondendo a uma biomassa aérea de $247 \mathrm{~g} / \mathrm{m}^{2}$ em uma área de $700 \mathrm{~m}^{2}$.

Não ocorreram diferenças significativas da biomassa de $A$. arenaria por área presente na zona A (comunidade psamófila-reptante) e na zona B da comunidade arbustiva de Palmae (Tabela 2). Porém, a biomassa por área da zona $\mathrm{A}$ diferiu significativamente das zonas $\mathrm{C}$ e $\mathrm{D}$. A zona $\mathrm{B}$ não diferiu da zona $\mathrm{C}$, mas sim da zona $\mathrm{D}$, e esta diferiu de todas as outras.

Foi encontrada uma relação positiva, altamente significativa $\left(F=39,57 ; \mathrm{R}^{2}=0,69 ; \mathrm{P}<0,01\right)$, entre a biomassa por área com distância do mar (Fig. 3).

A biomassa por indivíduo apresentou-se mais elevada nas depressões entre as cristas praiais (zonas B e D) do que em seus cumes (zonas A e C) (veja a Tabela 2). 


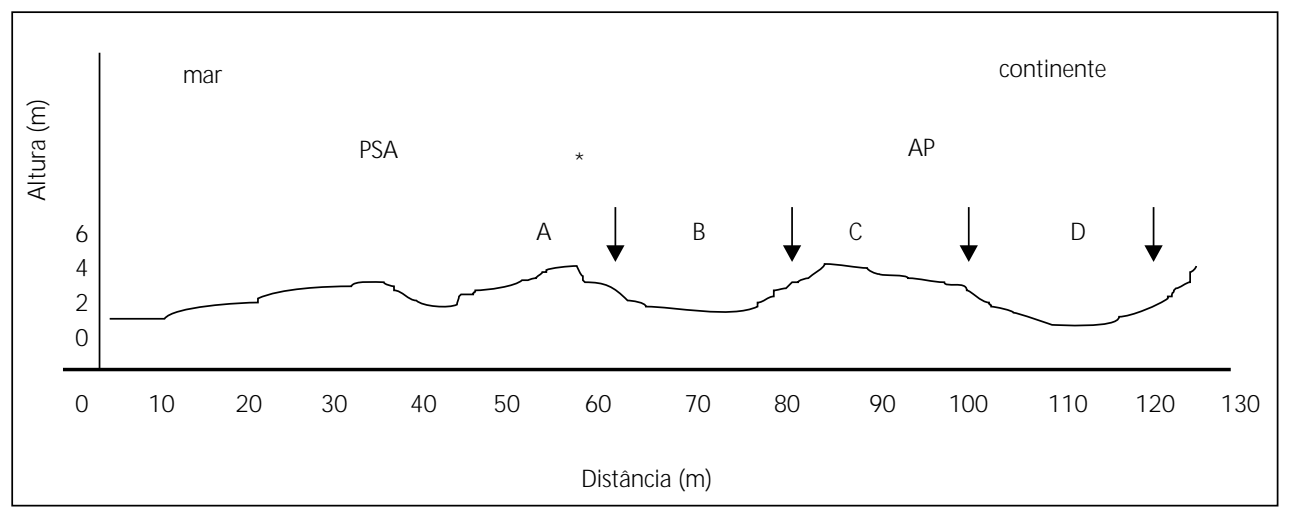

Fig. 2 - Diagrama esquemático representativo dos perfis topográficos e das zonas para a quantificação da bimassa aérea de Allagoptera arenaria no cordão externo da restinga de Marambaia, RJ. As zonas estão designadas como A, B, C e D. As setas indicam os limites entre as zonas de amostragem. O asterisco indica o limite entre as comunidades psamófila-reptante (PSA) e arbustiva de Palmae (PA).

TABELA 1

Análise comparativa da fertilidade e da granulometria dos solos em 3 zonas no sentido transversal ao cordão externo da restinga de Marambaia, RJ (zona $A$ = crista do primeiro cordão praial, zonas $B$ e $C=$ distantes, aproximadamente, 30 e $70 \mathrm{~m}$, respectivamente, da zona $A$ ).

\begin{tabular}{|c|c|c|c|}
\hline \multirow{2}{*}{ Parâmetros } & \multicolumn{3}{|c|}{ Zonas de Amostragem } \\
\cline { 2 - 4 } & $\mathbf{1}$ & $\mathbf{2}$ & $\mathbf{3}$ \\
\hline PH água 01:2,5 & 6,8 & 5,5 & 0,3 \\
\hline Al & 0,0 & 0,3 & 0,7 \\
\hline Ca + Mg meg/100 ml & 0,3 & 0,4 & 7 \\
\hline Na & 5 & 5 & 35 \\
\hline K ppm & 4 & 16 & 0,54 \\
\hline P ppm & 5 & 0,32 & 38 \\
\hline Carbono (\%) & 0,04 & 38 & 53 \\
\hline Areia grossa (\%) & 37 & 52 & 3 \\
\hline Areia fina (\%) & 55 & 4 & 6 \\
\hline Silte (\%) & 2 & 6 & \\
\hline Argila (\%) & 6 & & \\
\hline
\end{tabular}

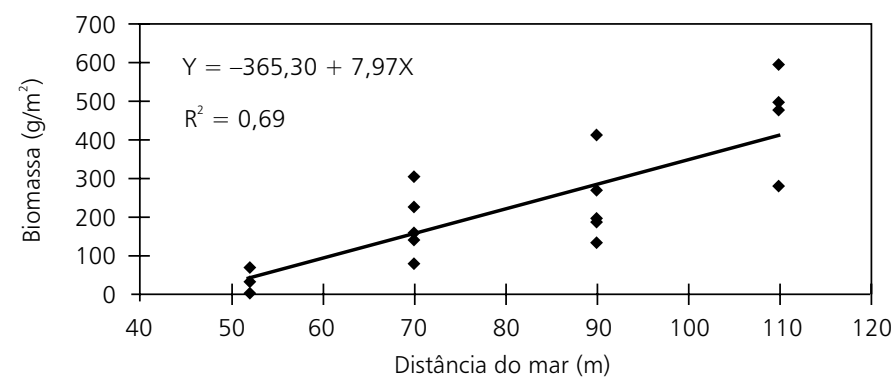

Fi. 3 - Relação entre a biomassa aérea de Allagoptera arenaria por área $\left(\mathrm{g} / \mathrm{m}^{2}\right)$ com a distância do mar $(\mathrm{m})$ na restinga de Marambaia, RJ. A linha representa a curva ajustada $(\mathrm{N}=20)$. 
TABELA 2

Distribuição das médias de biomassa aérea por área $\left(\mathrm{g} / \mathrm{m}^{2}\right)$, médias de biomassa aérea por indivíduo (g), médias das alturas e dos eixos de Allagoptera arenaria no sentido transversal ao cordão externo na restinga de Marambaia, RJ.

\begin{tabular}{|l|c|c|c|c|}
\hline \multirow{2}{*}{ Variáveis } & \multicolumn{4}{c|}{ Zonas do Cordão } \\
\cline { 2 - 5 } & $\mathbf{A}$ & B & C & D \\
\hline Biomassa/indivíduo (g)* & $7,93 \mathrm{a}$ & $12,50 \mathrm{~b}$ & $10,48 \mathrm{a}$ & $427,01 \mathrm{c}$ \\
\hline Biomassa/área $\left(\mathbf{g} / \mathbf{m}^{\mathbf{2}}\right)$ & $34,39 \mathrm{a}$ & $181,99 \mathrm{ba}$ & $0,96 \mathrm{~d}$ \\
\hline Altura (m) & $0,40 \mathrm{a}$ & $0,67 \mathrm{~b}$ & $241,58 \mathrm{~b}$ & $1,32 \mathrm{~d}$ \\
\hline Eixo (m) & $0,81 \mathrm{a}$ & $1,14 \mathrm{~b}$ & $0,59 \mathrm{c}$ & $1,02 \mathrm{c}$ \\
\hline
\end{tabular}

* Biomassa transformada por $\sqrt{\text { biomassa }}+0,5$.

As zonas B e D correspondem a depressões entre crista praiais. As zonas A (psamófila-reptante) e C correspondem aos cumes das cristas praiais

Médias seguidas de mesmas letras nas linhas não diferem pelo teste de Tukey a 5\%.

Não ocorreram diferenças significativas de biomassa por indivíduo entre as zonas A e C. Estas, por sua vez, diferiram significativamente das zonas $\mathrm{B}$ e D.

O desenvolvimento (médias de alturas e eixos) de $A$. arenaria variou de maneira similar às variações significativas encontradas na biomassa por indivíduos desta espécie, de acordo com a topografia da área.

As zonas $\mathrm{B}$ e $\mathrm{D}$, que corresponderam a depressões entre cristas praiais, apresentaram médias de altura e eixo de $A$. arenaria maiores que nas zonas A e C, que corresponderam aos cumes dessas cristas. A zona B, apesar de ter apresentado indivíduos de $A$. arenaria mais desenvolvidos em relação a zona $\mathrm{C}$, apresentou uma menor taxa média de biomassa por metro quadrado, porém não significativa (Tabela 2).

As médias das variáveis altura e eixo da copa de $A$. arenaria mostraram um padrão similar de variação ao longo do perfil topográfico, diferindose significativamente em todas as zonas (Tabela 2).

As médias da biomassa por indivíduo de $A$. arenaria aumentaram progressivamente do "transect" 1 ao 5. O "transect" 1 diferiu significativamente do "transect" 2 . Deste em diante, só ocorreram diferenças significativas entre as médias de maneira alternada (Tabela 3). No "transect" 1 ocorreu pouca variação da biomassa por indivíduo de $A$. arenaria ao longo das zonas, onde foi evidente a diferença entre as zonas C e D (Tabela 4).

TABELA 3

Médias das variáveis altura e eixo em cada "transect" perpendicular ao mar na restinga de Marambaia, RJ.

\begin{tabular}{|l|c|c|c|c|c|}
\hline \multirow{2}{*}{ Variáveis } & \multicolumn{5}{c|}{ "Transect" } \\
\cline { 2 - 6 } & $\mathbf{1}$ & $\mathbf{2}$ & $\mathbf{3}$ & $\mathbf{4}$ & $\mathbf{5}$ \\
\hline Altura (m) & $0,64 \mathrm{a}$ & $0,64 \mathrm{a}$ & $0,68 \mathrm{a}$ & $0,80 \mathrm{~b}$ & $0,88 \mathrm{~b}$ \\
\hline Eixo (m) & $0,99 \mathrm{c}$ & $1,08 \mathrm{dc}$ & $1,12 \mathrm{ad}$ & $1,24 \mathrm{ba}$ & $1,28 \mathrm{~b}$ \\
\hline Biomassa/indivíduo (g) & $9,56 \mathrm{a}$ & $11,47 \mathrm{~b}$ & $12,61 \mathrm{bc}$ & $13,50 \mathrm{~cd}$ & $14,72 \mathrm{~d}$ \\
\hline
\end{tabular}

* Biomassa transformada por $\sqrt{\text { biomassa }}+0,5$.

Médias seguidas de mesmas letras na coluna não diferem pelo teste Tukey a $5 \%$. 
TABELA 4

Distribuição das médias de biomassa aérea (g)* por indivíduo de Allagoptera arenaria nas zonas dos cinco "transects" analisados no sentido transversal ao cordão externo na Restinga de Marambaia, RJ.

\begin{tabular}{|c|c|c|c|c|}
\hline \multirow{2}{*}{ "Transect" } & \multicolumn{4}{|c|}{ Zonas } \\
\cline { 2 - 5 } & $\mathbf{A}$ & $\mathbf{B}$ & $\mathbf{C}$ & $\mathbf{D}$ \\
\hline $\mathbf{1}$ & $9,25 \mathrm{~A} \mathrm{ab}$ & $9,82 \mathrm{~A} \mathrm{ab}$ & $8,10 \mathrm{C} \mathrm{b}$ & $10,75 \mathrm{C} \mathrm{a}$ \\
\hline $\mathbf{2}$ & $7,24 \mathrm{~A} \mathrm{~b}$ & $13,66 \mathrm{~A} \mathrm{a}$ & $9,27 \mathrm{BC} \mathrm{b}$ & $14,14 \mathrm{~B} \mathrm{a}$ \\
\hline $\mathbf{3}$ & $3,67 \mathrm{~A} \mathrm{c}$ & $14,08 \mathrm{~A} \mathrm{a}$ & $6,36 \mathrm{ABC} \mathrm{b}$ & $15,47 \mathrm{~B} \mathrm{a}$ \\
\hline $\mathbf{4}$ & $10,64 \mathrm{~A} \mathrm{~b}$ & $10,75 \mathrm{~A} \mathrm{~b}$ & $12,40 \mathrm{AB} \mathrm{b}$ & $17,21 \mathrm{AB} \mathrm{a}$ \\
\hline $\mathbf{5}$ & $9,61 \mathrm{~A} \mathrm{~b}$ & $14,04 \mathrm{~A} \mathrm{~b}$ & $12,54 \mathrm{~A} \mathrm{~b}$ & $19,77 \mathrm{~A} \mathrm{a}$ \\
\hline
\end{tabular}

* Biomassa transformada por $\sqrt{\text { biomassa }}+0,5$.

Letras minúsculas comparam médias horizontais e letras maiúsculas comparam médias verticais. As médias seguidas de mesmas letras não diferem pelo teste Tukey a $5 \%$.

No "transect" 2, as zonas A e C diferiram das zonas B e D. No "transect" 3, a zona A diferiu de todas as outras. Já as zonas $\mathrm{A}, \mathrm{B}$ e $\mathrm{C}$ diferiram da zona D, no "transect" 4. O mesmo ocorreu no "transect" 5. Não houve diferenças significativas da biomassa por indivíduo entre as zonas A dos "transects" analisados. O mesmo foi verificado com a zona B. No entanto, as zonas C e D apresentaram pequenas diferenças entre os "transects" (veja a Tabela 4). A zona D foi a que apresentou maior depressão entre as cristas praiais e maior biomassa por indivíduo em todos os "transects", sendo a do "transect" 5 a de maior valor. Com exceção do "transect" 1 , em todos os outros, a zona A apresentou a menor biomassa por indivíduo de A. arenaria, sendo a do "transect" 3 a de menor valor.
As médias das alturas e eixos para cada "transect" estão representadas na Tabela 3. As médias das alturas nos "transects" 1,2 e 3 diferiram significativamente dos "transects" 4 e 5 , mostrando pouca variação. Quanto aos eixos, ocorreu um aumento das médias dos "transects" 1 ao 5 . A tendência do aumento não foi significativa para o "transect" imediatamente posterior, mas sim de maneira alternada.

Foi encontrada uma relação positiva, altamente significativa $\left(F=525,92 ; \mathrm{R}^{2}=0,92 ; \mathrm{P}<0,01\right)$, da cobertura de detritos com o distanciamento do mar (Fig. 4). A quantidade de detritos presente no trecho inicial da comunidade psamófila-reptante $(0-$ $30 \mathrm{~m}$ ) foi baixa e as variações não foram detectadas pelo método usado.

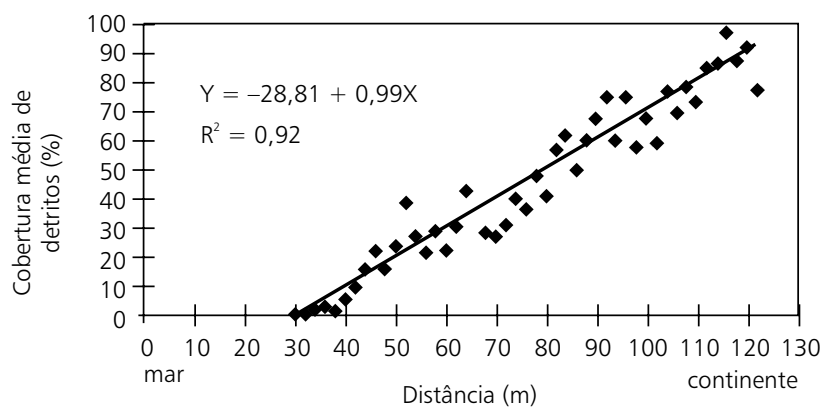

Fig. 4 - Relação entre a cobertura média de detritos (\%) nas comunidades psamófila-reptante e arbustiva de Palmae e o distânciamento do mar $(\mathrm{m})$, na restinga de Marambaia, RJ. A linha representa a curva ajustada $(\mathrm{N}=47)$. 


\section{DISCUSSÃO}

O sentido no qual ocorreu o aumento, tanto da biomassa aérea de $A$. arenaria quanto do incremento na cobertura de detritos no solo, na comunidade arbustiva de Palmae, coincide com o sentido apresentado por Menezes \& Araujo (1999) para o aumento do porcentual da cobertura vegetal dessa comunidade (mar-continente). Esses autores destacam que o aumento da cobertura vegetal dessa comunidade, nas zonas mais afastadas do mar, é devido a presença de $A$. arenaria.

$\mathrm{O}$ aumento na quantidade de detritos com o distanciamento do mar foi devido à contínua emissão de folhas em $A$. arenaria. Esta característica, comum nas palmeiras (Hallé et al., 1978), faz com que esta espécie apresente uma contínua senescênia deste tipo de material (Hay et al., 1982). A deposição e a subseqüente decomposição das folhas é o principal caminho para a transferência de carbono e nutrientes da planta para o solo (Vitousek, 1984; Vitousek \& Sanford, 1986; Gleeson \& Tilman, 1990; Nadkarni \& Mateison, 1992). Portanto, o aumento da quantidade de carbono com o distanciamento do mar representa a matéria orgânica em decomposição, provida principalmente das folhas de $A$. arenaria, que retornam ao solo os nutrientes absorvidos pela planta e que não foram lixiviados pelas chuvas para fora do sistema (Hay \& Lacerda, 1984; Conn \& Day, 1993).

A pouca variação da biomassa de $A$. arenaria por área com o distanciamento do mar pode ter sido influenciada por sua densidade. A menor quantidade de biomassa por área na crista praial próxima ao mar (zona $\mathrm{A}$ ), em relação àquela voltada para o interior (zona C), deve estar relacionada a um maior espaçamento entre os indivíduos observado naquela área, já que a biomassa por indivíduo não difere significativamente entre essas duas zonas. Contudo, a zona $\mathrm{C}$, embora topograficamente mais alta que a zona $\mathrm{A}$, apresenta médias de alturas e eixos das copas maiores que aquela zona. As condições que caracterizam os micro-hábitats nos ambientes de dunas frontais com o mar são mais estressantes do que aquelas que ocorrem nas dunas mais afastadas do mar. Estas condições referem-se à movimentação de areia pelo vento (Pfadenhauer, 1978; 1980; Costa et al., 1984), a ação do salsugem (Clayton, 1972; Barbour, 1978), a salinidade do solo (van der Valk, 1974; Holton \&
Johnson, 1979, Earle \& Kershaw, 1989), as altas temperaturas do ar e do solo (Oosting and Billings, 1942) e as flutuações no nível do lençol freático (Ranwell, 1959; Jones \& Etherington, 1971) que afetam o desenvolvimento da planta. Além disso, o solo na zona A é mais pobre, o que limita a produção de estruturas vegetativas e reprodutivas (Hay \& Lacerda, 1984, Schat et al., 1984). Por outro lado, subentendendo-se que a altura e o eixo da copa de $A$. arenaria são os responsáveis diretos pela biomassa aérea de cada indivíduo e que esta não diferiu entre as zonas $\mathrm{A}$ e $\mathrm{B}$, deveríamos esperar que as variáveis altura e eixo da copa também não fossem diferentes entre estas zonas, o que não ocorreu. Isso sugere que outras variáveis podem influenciar na biomassa aérea dessa espécie.

O menor desenvolvimento dessa espécie nos cumes das cristas praias pode estar relacionado com a topografia da área. Plantas nos cumes das cristas praiais são mais sujeitas ao estresse causado pelo salsugem e pelo vento, comparadas com aquelas que são mais desenvolvidas, localizadas nas depressões entre estas. Nessas depressões, as reservas nutritivas alocadas nas partes vegetativas são mobilizadas para a propagação assexuada por meio de rizomas, contribuindo para o adensamento da espécie nessas zonas e, conseqüentemente, o aumento da biomassa aérea.

Esse tipo de reprodução pode ser entendido como uma adaptação da planta para maximizar seus ganhos de energia em locais sobre estresse (Jurik \& Chabot, 1986; Voeks, 1988). O desenvolvimento da planta, desta forma, demonstra uma íntima relação com a topografia da área em questão. As touceiras de $A$. arenaria localizadas nas elevações do terreno (zonas A e C), caracterizadas por possuírem menor eixo da copa e altura, podem, possivelmente, serem afetadas pelo conteúdo de água no solo. Por estar mais distante do lençol freático, o desenvolvimento dessa espécie é influenciado por essa condição. Além disso, no cume das cristas praiais pode ocorrer uma maior lixiviação dos nutrientes, causando um empobrecimento nessas zonas, o que limita o crescimento da vegetação (Willis et al., 1959; van der Valk, 1975; Cordazzo, 1985). Nas depressões entre os cumes das cristas praiais, as condições para o desenvolvimento das plantas são melhores: ocorre uma maior proteção contra os ventos e o salsugem, diminuindo o estresse da planta e um maior acúmulo de matéria 
orgânica (Brown \& Lugo, 1982; Frangi \& Lugo, 1992).

A grande importância dessa matéria orgânica depositada no solo, consiste em sua capacidade de reter nutrientes, minimizando a lixiviação através do solo para fora da zona de raízes (Hay \& Lacerda, 1984; Kachi \& Hirose, 1983). A materia orgânica, sobre tudo, caracteriza-se como o principal sítio de troca catiônica em solos arenosos (Hay \& Lacerda, 1980). Além disso, há uma maior proximidade da zona das raízes com a água freática que funciona, não só como fonte, mas também como reservatório de nutrientes nos ecossistemas de restinga (Hay \& Lacerda, 1984).

As observações no campo mostraram que $A$. arenaria apresenta uma ampla rede de finas raízes, distantes entorno de $10 \mathrm{~cm}$ abaixo da superfície do solo e distante de outro indivíduo mais próximo 6 $\mathrm{m}$. Seu sistema de raízes finas e bastante ramificadas permite à planta reduzir a perda de nutrientes lixiviados no solo (Conn \& Day, 1993), favorecendo o sucesso dessas espécies nos ambientes de restinga impactados pelo fogo, por exemplo.

A tendência ao aumento progressivo da biomassa aérea de $A$. arenaria por "transect" observado na restinga de Marambaia, pode ser correlacionada com o aumento progressivo da sinuosidade dos perfis topográficos. Observações semelhantes foram feitas por Costa et al. (1988) nas dunas costeiras do Rio Grande do Sul, onde demonstraram que a distribuição espacial da biomassa aérea de Androtrichum trigynum correlacionava-se positivamente com a altura do lençol freático. Depressões mais acentuadas, além de proporcionarem mais chances ao acúmulo de matéria orgânica e protegerem as plantas da ação dos ventos e do salsugem, deixam o lençol freático mais próximo da superfície, possibilitando um maior desenvolvimento das plantas (Jones \& Etherington, 1971; Cordazzo, 1985; Cordazzo \& Seeliger, 1987).

A topografia dos seis perfis analisados por Menezes (1996) não foram semelhantes, sendo observado um ligeiro aumento na amplitude e na freqüência da sinuosidade, em direção do 1 ao 5 . Nessa mesma direção ocorreu o aumento da biomassa por indivíduo de $A$. arenaria, o que vem reafirmar a influência da topografia no desenvolvimento dessa espécie.

$\mathrm{O}$ acúmulo de folhas mortas de $A$. arenaria observado em torno de si, na zona mais afastada do mar, fornece uma maior quantidade de matéria orgânica para o solo. Assim, a presença de densas populações dessa espécie oferece um mecanismo para o enriquecimento do solo pelo aumento do conteúdo de matéria orgânica sob sua cobertura e, conseqüentemente, potencializa o acúmulo de nutrientes (Frangi \& Lugo, 1992). Nos solos pobres dos ecossistemas de restinga, particularmente na comunidade arbustiva de Palmae, A. arenaria pode ser apontada como o principal reservatório de nutrientes (Hay \& Lacerda, 1980, 1984), e a senescência de suas folhas, a principal forma de retornar ao solo parte dos nutrientes acumulados. Harper (1977) destaca a importância da estrutura populacional das plantas superiores por meio da quantificação de suas unidades modulares (isto é, folhas, flores, frutos etc.) e, para alguns propósitos, a dinâmica de partes da planta pode ser mais útil do que a dinâmica do próprio indivíduo na comunidade.

Dessa forma, as modificações no ambiente, principalmente pelo acúmulo de matéria orgânica no solo proveniente das folhas senescêntes de $A$. arenaria, podem caracterizar microclimas, propiciando o estabelecimento de outras espécies. Menezes \& Araujo (1999) apontam um maior valor de importância de nanofanerófitas $(\mathrm{VI}=24)$ na zona $\mathrm{D}$ em relação à zona $\mathrm{A}(\mathrm{VI}=0,2)$ nos "transects" analisados. Na primeira, ocorre uma maior biomassa aérea de $A$. arenaria, tanto por área quanto por indivíduo, em relação à segunda. Esta característica, além dos fatores físicos, pode estar relacionada às condições nutricionais do solo que apresenta-se mais fértil na zona mais afastada do mar.

Agradecimentos - Agradecemos a inestimada ajuda do Dr. Fabiano Mascarenhas Paulo Vicente pelo auxílio na análise estatística, ao Dr. John D. Hay pela revisão do manuscrito, aos biólogos Marlon da Costa Souza e Marcelo da Costa Souza pela colaboração nos trabalhos de campo e ao comando do Campo de Provas da Marambaia pela concessão para a realização deste estudo.

\section{REFERÊNCIAS BIBLIOGRÁFICAS}

ALMEIDA, A. L. \& ARAUJO, D. S. D., 1997, Comunidades vegetais do cordão arenoso externo da Reserva Ecológica Estadual de Jacarepiá, Saquarema, RJ. Oecol. Brasil, 3: 45-61.

ARAUJO, D. S. D. \& PEIXOTO, A. L., 1977, Renovação de uma comunidade vegetal de restinga após queimada. In: Congresso Nacional de Botânica, 27º, Rio de Janeiro. Anais. Acad. Bras. Ci., pp. 1-17. 
BARBOUR, M. G., 1978, Salt spray as a microenvironmental factor in the distribution of beach plants at Point Rays, California. Oecologia, Berl., 32: 213-224.

BROWN, S. \& LUGO, A., 1982, The storage and production of organic matter in tropical forest and their role in the global carbon cycle. Biotropica, 14: 161-187.

CARDOSO, M. S. R., 1995, Análise fitossociológica na formação Palmae de restinga no Parque Estadual Paulo César Vinha-Guarapari/ES. Monografia de Especialização, UFES, Vitória, 71p.

CIRNE, P. \& SCARANO, F. R., 1996, Rebrotamento após fogo de Andira legalis (Leguminosa) em restinga fluminense. In: H. S. Miranda, Sato \& B. F. S. Dias (orgs.), Impactos de queimadas em áreas de cerrado e restinga. UnB, pp. 128-136.

CLAYTON, J. L., 1972, Salt spray and mineral recycling in two California coastal ecosystems. Ecol., 53: 74-81.

CONN, C. E. \& DAY, F. P., 1993, Belowground biomass patterns on a coastal barrier island in Virginia. Bulletin of the Torrey Botanical Club, 120(2): 121-127.

CORDAZZO, C. V., 1985, Taxonomia e ecologia da vegetação das dunas costeiras ao sul de Cassino (RS). Tese de Mestrado, UFRG, Rio Grande, 103p.

CORDAZZO, C. V. \& COSTA, C. S. B., 1989, Associações vegetais das dunas frontais de Garopaba (SC). Ci. Cult. 41(9): 906-910

CORDAZZO, C. V. \& SEELIGER, U., 1987, Composição e distribuição da vegetação nas dunas costeiras do Rio Grande (RS). Ci. Cult., 39(3): 321-324.

CORDEIRO, S. Z., 1998, Análise da cobertura vegetal em três áreas de topografia distinta na praia do Peró, Cabo Frio, RJ. Tese de Mestrado, UFRJ, 78p.

COSTA, C. S. B., SEELIGER, U. \& CORDAZZO, C. V., 1984, Aspectos da ecologia populacional do Panicum racemosum Spreng. nas dunas costeiras do Rio Grande do Sul, Brasil. In: L. D. Lacerda, D. S. D. Araujo, R. Cerqueira \& B. Turqc (orgs.), Restingas: Origem, Estrutura, Processos. Niterói, CEUFF, pp. 395-411.

COSTA, C. S. B., SEELIGER, U. \& CORDAZZO, C. V., 1988, Dinâmica populacional de distribuição horizontal de Androtrichum trigynum (Spreng.) Pfeiffer (Cyperaceae) em brejos e dunas do Rio Grande do Sul, Brasil. Acta Limnol. Brasil., (11): 813-842.

EARLE, J. C. \& KERSHAW, K. A., 1989, Vegetation patterns in James Bay coastal marshes. III. Salinity and elevation as factors influencing plant zonations. Can. J. Bot., 67: 2967-2974.

FRANGI, J. L. \& LUGO, A. E., 1992, Biomassa and nutrient acumulation in ten year old bryophyte communities inside a flood plain in the Luquilo Experimental Forest, Puerto Rico. Biotropica, 24: 106-112.

GLEESON, L. K. \& TILMAN, D., 1990, Allocation and the transient dynamics of sucession on poor soils. Ecology, 71: 1144-1155.
HALlÉ, F., OLDEMAN, R. A. A. \& TOMLINSON, P. B. 1978, Tropical trees and forests: an architectural analysis. Springer-Verlang, 441p.

HARPER, J. L., 1977, Population biology of plants. Academic Press, London. 892p.

HAY, J. D., HENRIQUES, P. B. \& COSTA, S. R. A., 1982, Uma avaliação preliminar da possibilidade de usar equações de regressão para estimativas da biomassa na restinga. São Paulo, Rev. Brasil. Bot., 5: 33-36.

HAY, J. D. \& LACERDA, L. D. de., 1980, Alterações nas características do solo após a fixação de Neoregelia cruenta (R. Grah.) L. B. Smith (Bromeliaceae), em um ecossistema de restinga. Ci. Cult., São Paulo, 32(7): $\quad 863-867$.

HAY, J. D. \& LACERDA, L. D. de., 1984, Ciclagem de nutrientes no ecossistema de restinga. In: L. D. Lacerda, D. S. D. Araujo, R. Cerqueira \& B. Turcq (eds.), Restingas: Origem, Estrutura, Processos. CEUFF, Niterói, pp. 459-475.

HOLTON, B. Jr. \& JOHNSON, A. F., 1979, Dune scrub communities and their correlation with environmental factors at Point Reyes National Seashore, California. J. Biogeogr., 6: 317-328.

JONES, R. \& ETHERINGTON, J. R., 1971, The growth of dune and dune slack plants. J. Ecology, 59: 793-801.

JURIK, T. W. \& CHABOT, B. F., 1986, Leaf dynamics and profitability in wild strawberries. Oecol. (Berl.), 69: 296-307.

KACHI, N. \& HIROSE, T., 1983, Limiting nutrients for plant growth in coastal sand dune soils. J. Ecology, 71: 937-944

LACERDA, L. D., ARAÚJO, D. S. D. \& MACIEL, N. C., 1993, Dry coastal ecosystems. v. 2. Elsevier, Amsterdam.

LEITE, C. O., 1990, Biologia de reprodução de Allagoptera arenaria (Gomes) O. Kuntze (Diplothemium maritimum MART.) - Palmae, Rio de Janeiro. Tese de Mestrado, UFRJ, 80p.

MENEZES, L. F. T., 1996, Caracaterização de comunidades vegetais praianas da Restinga da Marambaia, RJ. Tese de Mestrado, UFRRJ, 89p.

MENEZES, L. F. T. \& ARAUJO, D. S. D., 1999 Estrutura de duas formações vegetais do cordão externo da restinga de Marambaia, RJ. Acta Bot. Bras., 13(2): 223-235.

NADKARNI, N. M. \& MATEISON, T. J., 1992, Biomass and nutrient dynamics of fine litter of terrestrially rooted material in a neotropical montane forest, Costa Rica. Biotropica, 24: 113-120.

OOSTING, H. J. \& BILlingS, W. D., 1942, Factors affecting vegetational zonation on coastal dunes. Ecol., 23(2): 131-142.

PACKHAM, J. R. \& WILLIS, A. J., 1997, Ecology of dunes, salt marsh and shingle. Chapman e Hall. London, $335 \mathrm{p}$. 
PFADENHAUER, J., 1978, Contribuição ao conhecimento da vegetação e de suas condições de crescimento nas dunas costeiras do Rio Grande do Sul, Brasil. Rev. Brasil. Biol., 38: 827-836.

PFADENHAUER, J., 1980, Die vegetation der kustendunen von Rio Grande do Sul, Sul Brasiliens. Phytoecoen, 8: 321-364.

RANWELL, D. S., 1959, Newborough warren, anglesey. I. The dune system and dune slack habitat. J. Ecol., 47: 571-601.

RAJPUROHIT, K. S. \& Sen, D. N., 1977, Soil salinity and seed germination under water stress. Trans. Isdt. Ucds., 2(1): 106-110.

RIZZINI, C. T., 1979, Tratado de fitogeografia do Brasil: aspectos sociológicos e florísticos. v. II. HUCITECEDUSP, 374p.

SCHAT, H. A., BOS, A. H. \& SCHOLTEN, M., 1984, The mineral nutrition of some therophytes from oligotrophic dune slack soil. Acta Oecol./Plant., 5: 129131.

TOMLINSON, P. B., 1960, Essays on the morphology of palms. II. The early growth of the palm. Principes, 4(4): 140-143. van der VALK, A. G., 1974, Mineral cycling in coastal foredune plant communities in Cape hatteras National Seashore. Ecol., 55: 1349-1358.

van der VALK, A. G., 1975, The floristic composition and structure of foredune plant communities of Cape Hatteras National Seashore. Cheas. Scien., 16(2): 115126 .

VITOUSEK, P., 1984, Nutrient cycling and nutrient use efficiency. Am. Nat., 119: 553-572.

VITOUSEK. P. \& Sanford, R., 1986, Nutrient cycling in moist tropical forest. Ann. Rer. Ecol. Syst., 17: 137167.

VOEKS, R. A., 1988, Changing sexual expression of a Brazilian rain forest palm (Attalea funifera Mart.) Biotropica, 20: 107-113.

WILLIS, A. J., Hope-Simpson, B. F. \& Yemm, E. W., 1959, Brauton Burrows the dunesystem and its vegetation. Part I. J. Ecol., 47: 1-24. 